time of germination are rarely attacked afterwards. If the necrosis does not extend to the cotyledons, the seedling may recover due to the development of axillary buds. This type of recovery occurs among plants infected towards the end of their period of susceptibility, that is, 5-6 weeks after germination, and particularly when dry conditions prevail during the period immediately following infection.

Sporangia generally appear on the petiole and upper part of the stem 5-6 days after infection, although in a few instances they have been observed after 4 days. The pathogen produces sporangia in culture on potato dextrose agar similar to those of $P$. palmivora, but of smaller dimensions. It is thought that the causal a.gent of seedling die-back may be a form of $P$. palmivora; but isolates are at present being examined at the Commonwealth Mycological Institute with the view of specific identification.

A large measure of control (95 per cent) has been achieved using a proprietary copper fungicide. However, it was necessary to spray at frequent intervals ( 3 or 6 days) as only a low level of control was obtained with less-frequent (9 or 12 days) applications. The control experiments are being continued and will be fully reported elsewhere.

Federal Department of Agricultural

S. R. Chant Research Ibadan, Nigeria.

1 Orellana, R. G. Tenth Ann. Rep. Inter-American Institute of Agricultural Sciences, Turrialba, Costa Rica, 1952 (1953). ${ }^{2}$ Van Suchtelen, N. J., Surinaam Landb., 3, 3, 223 (1955).

* Thorold, C. A., Nature, 170, 718 (1952)

\section{A New Technique for the Study of Laboratory Populations of Drosophila}

Investigation of the relationships between popula. tion size and gene frequency in laboratory studies of Drosophila have been limited to small breeding groups (usually of less than one thousand individuals) because of the difficulty of estimating population number. Direct counts can be made when units of the types described by Reed and Reed ${ }^{1}$ and BuzzatiTraverso ${ }^{2}$ are in use, but the environment in these systems lacks constancy. The former has been modified $^{3,4}$ for work on larger populations; but with this design there remains the possibility that migration between sections of the cage might be somewhat restricted. For that reason it was felt that the following technique might be of interest to other workers in this field.

The cages in operation at the present time (Fig. 1) are constructed of light gauge galvanized iron. Three rectangular section wire mesh channels which carry the food dishes run lengthwise between the doors at either end. The bottom of the cage is formed by an inverted pyramid terminating beneath in a screw connexion fitting a small opaque glass jar. Ventilation is provided by wire gauze panels in the sides. The top is of glass. The covers of the feeding ports are secured by wing-nuts, cork gaskets ensuring that they fit tightly against the cage.

Discarded food dishes are removed from one end of the cage as the fresh ones are pushed in at the other, erch dish remaining in the cage for a time considerably in excess of the life-cycle of the flies. One, two or three food channels are used in the cage according to the population density required. Channels not in use for food carry dishes containing cellulose tissue

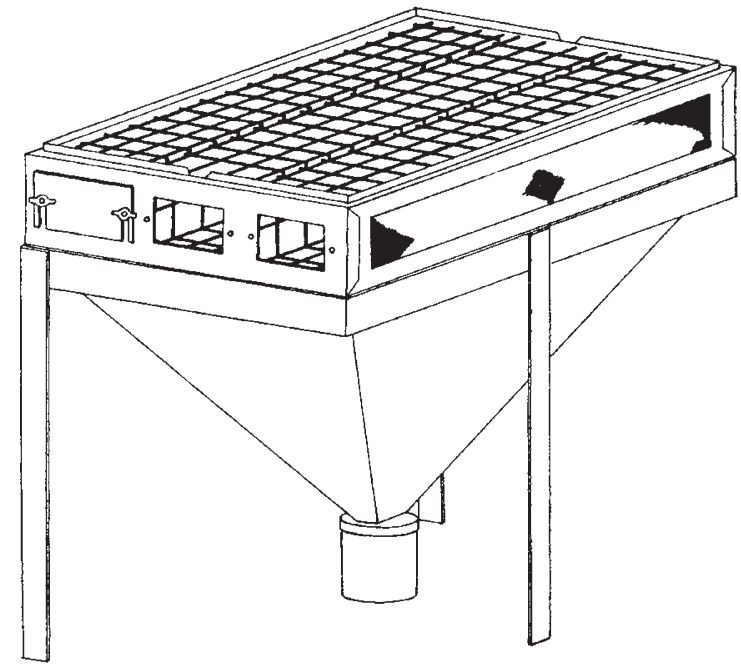

Fig. 1. The population cage (axometric projection)

soaked in water so that comparable humidities are maintained in all cages. Uniformity of humidity within the units is ensured by reversing the direction of movement of the food or water dishes along alternate channels.

Population counts are made fortnightly. Debris is first dislodged by tapping, and the glass jar fitted to the bottom of the cage is cleaned out. Carbon dioxide is then admitted, achieving almost instantaneous anæsthesia, and the flies drop into the jar. Any lodged in crevices or on the surfaces of the food dishes are removed by the blast of gas. The jar is unscrewed from the cage and the flies are shaken out evenly on to sheets of 'Kodagraph' contact copying paper in the darkroom and a brief exposure made. Random samples are taken for scoring gene frequencies, and are returned to the cage with the rest of the population. Provided that the flies are well spread out during the few minutes needed for recovery, there is no mortality. As the flies are readily dis. tinguishable from any debris which may have fallen into the collecting jar with them, the determination of population size is made directly from the photographic record.

Food dishes and adult flies may be transferred to a clean cage as this becomes necessary. In the event of mite infestation, monthly transfers afford satisfactory control, eliminating the use of chemical deterrents ${ }^{5}$ of doubtful safety.

In the experiments being conducted in cages of this type, the growth of the population is at first rapid and oscillation of size extreme. These fluctuations continue about an equilibrium density but, over the present experimental period, gradually decrease in amplitude. Population growth and the rate of elimination of marker alleles depend upon the genetic heterogeneity of the population as well as on its size. The results of this work will be reported in detail elsewhere.

\section{J. A. Thomson}

Zoology Department,

University of Melbourne. Aug. 29.

1 Reed, S. C., and Reed, E. W., Genetics, 33, 121 (1949).

2 Buzzati-Traverso, A. A., Heredity, 9, 153 (1955).

${ }^{3}$ Da Cunha, A. B., Evolution, 3, 239 (1949).

- Erk, F. C., Genetics, 40, 331 (1955).

"Ludwin, I., Evolution, 5, 231 (1951). 